\title{
Herpes Simplex Virus is Akt-ing in translational control
}

\author{
Kara L. Norman and Peter Sarnow ${ }^{1}$ \\ Department of Microbiology and Immunology, Stanford University School of Medicine, Stanford, California 94305, USA
}

\begin{abstract}
All viruses depend on the cellular protein synthesis machinery for the production of viral proteins. Thus, viruses have evolved a variety of strategies to avoid innate host responses that inhibit protein synthesis. In this issue of Genes \& Development, Chuluunbaatar and colleagues (pp. 2627-2639) demonstrate that Herpes Simplex Virus-1 counteracts this response through viral kinase Us3, which mimics cellular kinase Akt to phosphorylate and repress tuberous sclerosis complex 2 (TSC2), resulting in the activation of mammalian target of rapamycin complex 1 (mTORC1) and enhancement of mRNA translation.
\end{abstract}

Translation initiation is the rate-limiting step in protein biosynthesis and is controlled by at least two major mechanisms (Sonenberg and Hinnebusch 2009). One mechanism relies on the abundance of functional initiator tRNA molecules bound to eukaryotic initiation factor 2 (eIF2). For example, when eukaryotic cells encounter heat-induced protein misfolding, nutrient starvation, or virus infection, the $\alpha$ subunit of trimeric eIF2 is phosphorylated by a variety of protein kinases. As a result, formation of ternary initiator tRNA-eIF2 complexes becomes limiting and translation initiation at the AUG start codon is inefficient (Sonenberg and Hinnebusch 2009). A second mechanism is dependent on the phosphorylation status of the 4E-binding proteins (4E-BPs). In particular, dephosphorylated 4E-BP binds the cap-binding protein eIF4E, competing with eIF4G for eIF4E. Interaction of eIF4E with eIF4G is important in the formation of the multicomponent complex eIF4F, which recruits the 40S ribosomal subunits to the mRNA (Fig. 1). Thus, sequestration of eIF4E by 4E-BP inhibits cap-dependent translation initiation (Sonenberg and Hinnebusch 2009).

Role of the mammalian target of rapamycin complex (mTORC) signaling pathway in translational control

As predicted, 4E-BPs are hyperphosphorylated in actively dividing cells when cap-dependent translation is very

[Keywords: Translational control; herpesvirus replication; viral kinase; mTOR activation; Akt signaling]

${ }^{1}$ Corresponding author.

E-MAIL psarnow@stanford.edu; FAX (650) 498-7147.

Article is online at http://www.genesdev.org/cgi/doi/10.1101/gad.2004510. efficient. There was much excitement when mTOR was identified as the kinase that phosphorylates the 4E-BPs and the ribosomal S6 kinase (S6K) (Ma and Blenis 2009). mTORC is regulated by the heterodimeric tuberous sclerosis complex (TSC1/2) and the compound rapamycin (Ma and Blenis 2009). For example, when growth factors are abundant, translation is promoted because TSC $1 / 2$ is inactivated by phosphorylation by serine/threonine protein kinases Akt or ERK (Fig. 1). TSC1/2 inactivation leads to accumulation of Ras homology-enriched in brain small G protein (Rheb)-GTP (Tee et al. 2003), which stimulates mTORC kinase activity, leading to the accumulation of phosphorylated 4E-BP and formation of eIF4E-eIF4G complexes (Fig. 1). In contrast, when ATP/AMP ratios are low, translation is inhibited because AMP-activated protein kinase AMPK activates TSC2 (Inoki et al. 2003), which then acts as a GTPase-activating protein to promote GTP hydrolysis by Rheb (Tee et al. 2003). Rheb-GDP accumulation inactivates mTORC, leading to the accumulation of hypophosphorylated 4E-BP and sequestration of eIF4E. Overall, mTORC regulates the efficiency of capdependent translation by phosphorylation of 4E-BP (Fig. 1).

Interference of herpesviruses with the mTORC signaling pathway

Herpesviruses can modulate the TSC/mTORC/4E-BP/ eIF4E signaling cascade in several ways to ensure continuous viral protein synthesis in infected cells. For example, human cytomegalovirus (HCMV) encodes UL38, which is a TSC2-binding protein that promotes mTORC activation (Moorman et al. 2008). Epstein-Barr virus encodes latency protein LMP2A, which activates Akt and mTORC, leading to 4E-BP phosphorylation and translation activation (Moody et al. 2005). Finally, Kaposi's sarcoma-associated herpesvirus expresses a viral G protein-coupled receptor (VGPCR) that constitutively activates Akt, leading to phosphorylation and inactivation of TSC2 (Sodhi et al. 2006). Yet, the role that mTORC plays during Herpes Simplex Virus-1 (HSV-1) infection has remained a relatively unexplored area.

The current study by Chuluunbaatar et al. (2010) reveals a novel, surprising mechanism by which HSV-1 modulates the phosphorylation of 4E-BPs by an Akt mimetic protein. First, Chuluunbaatar et al. (2010) noted that expression of nonphosphorylatable 4E-BP, a translational 


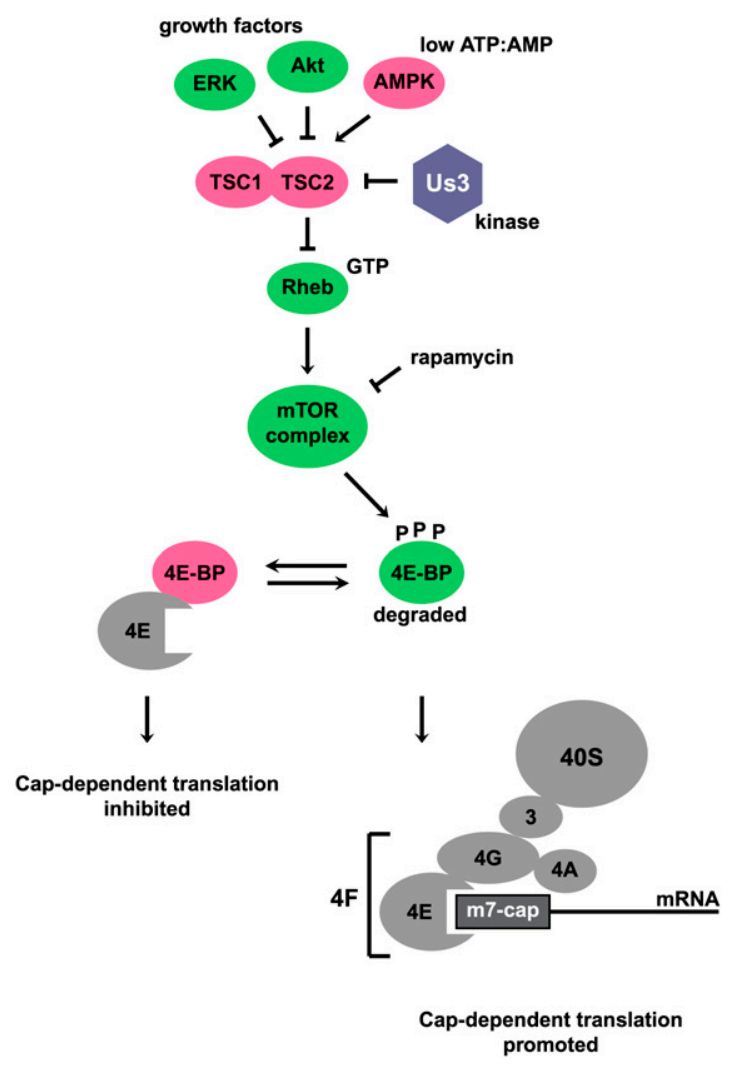

Figure 1. Modulation of the mTORC pathway by HSV-1. See the text for details. Factors promoting cap-dependent translation initiation are shown in green. Proteins that inhibit cap-dependent translation are shown in pink. The translational machinery is shown in gray, and viral protein Us3 is shown in blue.

repressor (Fig. 1), diminished viral protein synthesis. This finding suggested that accumulation of phosphorylated 4E-BP, a substrate of mTORC, was necessary to augment HSV-1 mRNA translation (Fig. 1).

\section{Searching for HSV-1-induced kinases that mediate 4E-BP phosphorylation}

HSV-1 expresses two serine/threonine protein kinases: UL13 and Us3. Therefore, it was possible that one of these kinases regulated signaling in the mTORC pathway. While UL13-deficient HSV-1 was still able to induce 4EBP1 phosphorylation, deletion of the viral Us3 protein kinase gene resulted in a marked increase in the hypophosphorylated form of 4E-BP1. Reintroduction of the Us3 gene rescued 4E-BP1 phosphorylation in recombinant virus-infected cells. Reintroduction of an Us3 mutant gene with a defect in the Us3 kinase domain, however, failed to rescue the phenotype, demonstrating that the enzymatic activity of Us3 was needed to signal 4EBP1 hyperphosphorylation.

\section{Identification of the Us3 kinase substrate in the mTORC signaling pathway}

HSV-1 has been shown to activate Akt (Benetti and Roizman 2006), which could potentially activate the phosphorylation of 4E-BP1 by mTORC through TSC2. However, Chuluunbaatar et al. (2010) found that specific inhibition of Akt did not block wild-type HSV-1-induced 4E-BP1 phosphorylation. Curiously, the Akt phosphorylation sites in TSC2, at positions T1462 and S939, were phosphorylated in HSV-1-infected cells. These findings suggest that a kinase other than Akt phosphorylated TSC2 at positions T1462 and S939. Remarkably, deletion of the Us3 gene or elimination of Us3 kinase activity abrogated HSV-1-stimulated TSC2 phosphorylation, arguing that Us3 or an Us3-induced kinase phosphorylated TSC2.

Us3 shares little similarity with Akt, yet Chuluunbaatar et al. (2010) presented three lines of evidence demonstrating that Us3 is likely a functional Akt surrogate. First, TSC2 was phosphorylated when Us3 was expressed in isolation, independently of HSV-1 infection. Second, in vitro kinase assays using immunopurified Us3 and TSC2 showed that TSC2 phosphorylation depended on Us3 kinase activity and ATP. Last, direct phosphorylation of TSC2 by the viral kinase was shown using engineered Us3 that can use a bio-orthogonal ATP $\gamma \mathrm{S}$ analog (Allen et al. 2007). In this remarkable technique, a kinase is engineered to specifically use $\mathrm{N}^{6}$-alkylated ATP $\gamma \mathrm{S}$ to thiophosphorylate substrates. Unengineered kinases cannot use the alkylated thiolated ATP. Targets of the engineered kinase are subsequently detected in Western blots using antibodies directed against thiophosphateester moieties (Allen et al. 2007). Indeed, only engineered Us3 could thiophosphorylate TSC2, providing excellent support that TSC2 is a direct target of the Us3 kinase. To determine whether TSC2 phosphorylation ultimately affected 4E-BP1, a nonphosphorylatable, constitutively active TSC2 was expressed that prevented HSV-1-induced 4E-BP1 phosphorylation. This finding suggests that Us3 phosphorylates and inhibits TSC2 activity to allow phosphorylation of 4E-BP1.

To examine the biological consequences of Us3mediated TSC2 phosphorylation, Chuluunbaatar et al. (2010) examined the effects of an Us3 gene deletion on viral growth. Indeed, the Us3 deletion mutant displayed diminished virus production. To determine whether this was due to impaired TSC2 inhibition, TSC2 was depleted by siRNAs. Strikingly, TSC2 depletion rescued the growth of the Us3-deleted virus by 20 -fold. These data provide genetic evidence that Us3-mediated inhibition of TSC2 allows for efficient HSV-1 virus production. Nonetheless, it will be interesting to learn whether viral growth is specifically enhanced at the level of viral mRNA translation, or whether ongoing cellular translation is also required.

\section{Us3 phosphorylates several cellular substrates of Akt}

Chuluunbaatar et al. (2010) examined whether Us3 could also phosphorylate known cellular Akt substrates. They provided evidence that Akt substrates FOXO1 and GSK3 were phosphorylated by the viral kinase. Thus, it will be interesting to explore whether phosphorylation of FOXO1, GSK3, and other cellular substrates influences 
the outcome of HSV-1 infection. In this respect, it is noteworthy that depletion of TSC2 rescued the deleted Us3 virus by only 20 -fold, but not to wild-type levels.

\section{Viral strategies to usurp the cellular translation apparatus}

As mentioned above, many virus infections induce the phosphorylation of eIF2, which results in the inhibition of both viral and cellular mRNA translation. Thus, viruses have evolved elaborate ways to down-regulate the eIF2 kinases. In this Perspective, we briefly summarize how viruses can modulate the mTORC pathway and the activity of the cap-binding protein complex to ensure access of the viral mRNAs to the translation apparatus (Fig. 2). We refer the interested reader to an excellent recent review on this topic (Roberts et al. 2009).

Poxviruses and adenoviruses activate the mTORC pathway to enhance 4E-BP phosphorylation and stimulate cap-dependent mRNA translation (Roberts et al. 2009). Similarly, human papillomavirus (HPV) also interferes with the mTORC pathway by a mechanism in which the HPV16 E6 oncoprotein binds and induces the degradation of TSC2 (Fig. 2; Lu et al. 2004). However, a recent study by Spangle and Munger (2010) did not observe an HPV16-induced TSC2 degradation. Rather, Spangle and Munger (2010) noted an enhanced Akt activity, which stimulated mTORCl and led to a rapamycin-sensitive increase in cap-dependent translation. Picornaviruses and rhabdoviruses, on the other hand, can induce the dephosphorylation of 4E-BPs, leading to inhibition of translation (Roberts et al. 2009).

Most viral mRNAs are capped, and must therefore compete with capped, cellular mRNAs for the cap-binding protein complex eIF4F (Figs. 1, 2). Herpesvirus and adenovirus infections modulate the Mnk kinase that phosphorylates the cap-binding protein eIF4E, enhancing its affinity for cap structures. Several DNA and RNA viruses modify or manipulate the eIF4F scaffolding protein eIF4G (Fig. 2). For example, several picornaviruses proteolyze eIF4G and the poly(A)-binding protein, which results in the inhibition of translation of capped mRNAs. However, most picornaviral mRNAs can be translated by an internal ribosome entry mechanism that does not require eIF4E (Roberts et al. 2009). Interestingly, infection of insect cells with cricket paralysis virus results in the dissociation of eIF4G from eIF4F (Garrey et al. 2010), resulting in the inhibition of host mRNA translation, but allowing viral mRNA translation by an internal ribosome entry mechanism.

Other RNA viruses have found creative ways to usurp or circumvent cap-dependent regulation during infection, and we point out two astonishing examples. Influenza virus is known to employ a "cap-snatching" mechanism in which $5^{\prime}$-terminal $\mathrm{m}^{7} \mathrm{G}$-capped RNA fragments are "stolen" from cellular mRNAs and attached to the viral mRNAs (Boivin et al. 2010). Hantavirus nucleocapsid $\mathrm{N}$ protein is a cap-binding protein that substitutes for all three members of the eIF4F cap-binding complex (Mir and Panganiban 2008). Thus, the $\mathrm{N}$ protein is predicted to facilitate cap-dependent translation when 4E-BPs are dephosphorylated.

\section{Concluding remarks}

While all of these examples point to sophisticated mechanisms by which viruses can hijack ribosomes from the host, they also point to viral Achilles' heels. It is logical to invoke the mTORC pathway as an antiviral target to combat HSV-1. However, most inhibitors of mTORC also have immunosuppressive effects. Thus, targeting viral Us3 seems to be an attractive idea, especially if specific Us3 modulators do not affect normal functions of Akt. Of course, it needs to be seen whether Us3 has other
A.

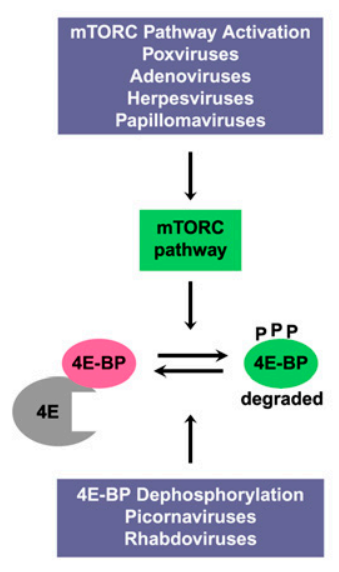

B.

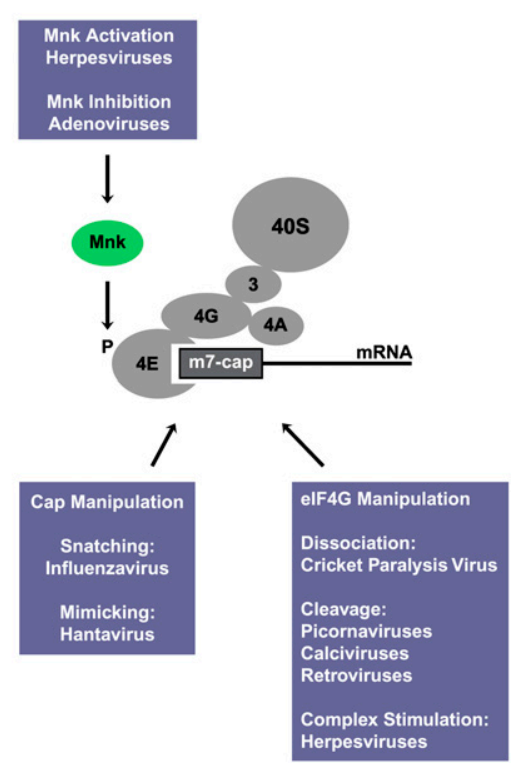

Figure 2. Modulation of the mTORC pathway $(A)$ and the cap-binding protein complex $(B)$ by viruses. See the text for details. 
hitherto undetected functions that may affect virus-host pathogenesis.

\section{Acknowledgments}

We thank Cara Pager for critical reading of the manuscript. K.L.N. is supported by a Fellowship from the Alberta Heritage Foundation for Medical Research. P.S. is funded by the National Institutes of Health.

\section{References}

Allen JJ, Li M, Brinkworth CS, Paulson JL, Wang D, Hubner A, Chou WH, Davis RJ, Burlingame AL, Messing RO, et al. 2007. A semisynthetic epitope for kinase substrates. Nat Methods 4: 511-516.

Benetti L, Roizman B. 2006. Protein kinase B/Akt is present in activated form throughout the entire replicative cycle of $\Delta \mathrm{U}(\mathrm{S}) 3$ mutant virus but only at early times after infection with wild-type herpes simplex virus 1. J Virol 80: 3341-3348.

Boivin S, Cusack S, Ruigrok RW, Hart DJ. 2010. Influenza A virus polymerase: Structural insights into replication and host adaptation mechanisms. J Biol Chem 285: 28411-28417.

Chuluunbaatar U, Roller R, Feldman ME, Brown S, Shokat KM, Mohr I. 2010. Constitutive mTORC1 activation by a herpesvirus Akt surrogate stimulates mRNA translation and viral replication. Genes Dev (this issue). doi: 10.1101/gad.1978310.

Garrey JL, Lee YY, Au HH, Bushell M, Jan E. 2010. Host and viral translational mechanisms during cricket paralysis virus infection. J Virol 84: 1124-1138.

Inoki K, Zhu T, Guan KL. 2003. TSC2 mediates cellular energy response to control cell growth and survival. Cell 115: 577590.

Lu Z, Hu X, Li Y, Zheng L, Zhou Y, Jiang H, Ning T, Basang Z, Zhang C, Ke Y. 2004. Human papillomavirus 16 E6 oncoprotein interferences with insulin signaling pathway by binding to tuberin. J Biol Chem 279: 35664-35670.

Ma XM, Blenis J. 2009. Molecular mechanisms of mTORmediated translational control. Nat Rev Mol Cell Biol 10: 307-318.

Mir MA, Panganiban AT. 2008. A protein that replaces the entire cellular eIF4F complex. EMBO J 27: 3129-3139.

Moody CA, Scott RS, Amirghahari N, Nathan CO, Young LS, Dawson CW, Sixbey JW. 2005. Modulation of the cell growth regulator mTOR by Epstein-Barr virus-encoded LMP2A. J Virol 79: 5499-5506.

Moorman NJ, Cristea IM, Terhune SS, Rout MP, Chait BT, Shenk T. 2008. Human cytomegalovirus protein UL38 inhibits host cell stress responses by antagonizing the tuberous sclerosis protein complex. Cell Host Microbe 3: 253-262.

Roberts LO, Jopling CL, Jackson RJ, Willis AE. 2009. Viral strategies to subvert the mammalian translation machinery. In Progress in molecular biology and translational science, vol. 90: Translational control in health and disease (ed. JWB Hershey), pp. 313-367. Elsevier, London. doi: 10.1016/S18771173(09)90009-6.

Sodhi A, Chaisuparat R, Hu J, Ramsdell AK, Manning BD, Sausville EA, Sawai ET, Molinolo A, Gutkind JS, Montaner S. 2006. The TSC2/mTOR pathway drives endothelial cell transformation induced by the Kaposi's sarcoma-associated herpesvirus G protein-coupled receptor. Cancer Cell 10: 133143.

Sonenberg N, Hinnebusch AG. 2009. Regulation of translation initiation in eukaryotes: Mechanisms and biological targets. Cell 136: 731-745.
Spangle JM, Munger K. 2010. The human papillomavirus type 16 E6 oncoprotein activates mTORC1 signaling and increases protein synthesis. I Virol 84: 9398-9407.

Tee AR, Manning BD, Roux PP, Cantley LC, Blenis J. 2003. Tuberous sclerosis complex gene products, Tuberin and Hamartin, control mTOR signaling by acting as a GTPaseactivating protein complex toward Rheb. Curr Biol 13: 12591268. 


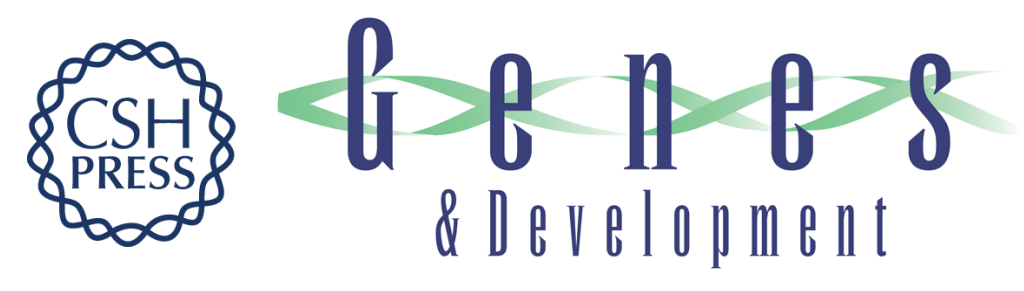

\section{Herpes Simplex Virus is Akt-ing in translational control}

Kara L. Norman and Peter Sarnow

Genes Dev. 2010, 24:

Access the most recent version at doi:10.1101/gad.2004510

Related Content Constitutive mTORC1 activation by a herpesvirus Akt surrogate stimulates mRNA translation and viral replication

Uyanga Chuluunbaatar, Richard Roller, Morris E. Feldman, et al.

Genes Dev. December, 2010 24: 2627-2639 Mimicry in Akt-ion

Annalisa M. VanHook

Sci. Signal. December , 2010 3: ec386

References This article cites 14 articles, 6 of which can be accessed free at:

http://genesdev.cshlp.org/content/24/23/2583.full.html\#ref-list-1

Articles cited in:

http://genesdev.cshlp.org/content/24/23/2583.full.html\#related-urls

License

Email Alerting Receive free email alerts when new articles cite this article - sign up in the box at the top Service right corner of the article or click here.

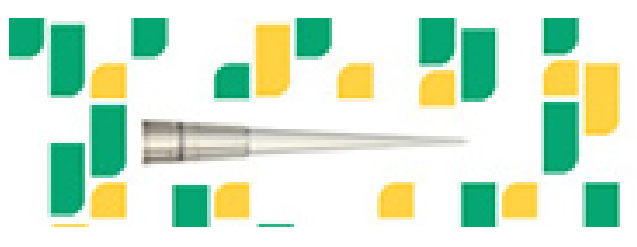

Focused on your science. 